\title{
The effect of intravenous golimumab on health-related quality of life and work productivity in patients with active psoriatic arthritis: results of the Phase 3 GO-VIBRANT trial
}

\author{
Alexis Ogdie ${ }^{1}$ (D) - Jessica A. Walsh ${ }^{2}$ - Soumya D. Chakravarty ${ }^{3,4}$ (D) Steven Peterson $^{5} \cdot$ Kim Hung Lo $^{6} \cdot$ Lilianne Kim $^{6}$. \\ Nan $\mathrm{Li}^{7}$ • Elizabeth C. Hsia ${ }^{1,6}$. Eric K. H. Chan ${ }^{7}$ - Arthur Kavanaugh ${ }^{8}$ - M. Elaine Husni ${ }^{9}$
}

Received: 29 June 2020 / Revised: 3 February 2021 / Accepted: 4 February 2021 / Published online: 2 March 2021

(C) The Author(s) 2021

\begin{abstract}
Introduction/objectives To evaluate changes in health-related quality of life (HRQoL) and productivity following treatment with intravenous (IV) golimumab in patients with psoriatic arthritis (PsA).

Methods Patients were randomized to IV golimumab $2 \mathrm{mg} / \mathrm{kg}(n=241)$ at Weeks 0 , 4, then every 8 weeks (q8w) through Week 52 or placebo $(n=239)$ at Weeks 0,4 , then q8w, with crossover to IV golimumab $2 \mathrm{mg} / \mathrm{kg}$ at Weeks 24,28 , then q8w through Week 52. Change from baseline in EuroQol-5 dimension-5 level (EQ-5D-5L) index and visual analog scale (EQ-VAS), daily productivity VAS, and the Work Limitations Questionnaire (WLQ) was assessed. Relationships between these outcomes and disease activity and patient functional capability were evaluated post hoc.

Results At Week 8, change from baseline in EQ-5D-5L index (0.14 vs 0.04), EQ-VAS (17.16 vs 3.69), daily productivity VAS $(-2.91$ vs -0.71$)$, and WLQ productivity loss score $(-2.92$ vs -0.78$)$ was greater in the golimumab group versus the placebo group, respectively. At Week 52, change from baseline was similar in the golimumab and placebo-crossover groups (EQ-5D-5L index: 0.17 and 0.15; EQ-VAS: 21.61 and 20.84; daily productivity VAS: -2.89 and -3.31 ; WLQ productivity loss: -4.49 and -3.28 , respectively). HRQoL and productivity were generally associated with disease activity and functional capability, with continued association from Week 8 through Week 52.

Conclusion IV golimumab resulted in early and sustained improvements in HRQoL and productivity from Week 8 through 1 year in patients with PsA. HRQoL and productivity improvements were associated with improvements in disease activity and patient functional capability.

\section{Key Points}

- In patients with active psoriatic arthritis (PsA), intravenous (IV) golimumab improved health-related quality of life (HRQoL) and productivity as early as 8 weeks and maintained improvement through 1 year

- Improvements in HRQoL and productivity outcomes in patients with PsA treated with IV golimumab were associated with improvements in disease activity and patient functional capability outcomes

- IV golimumab is an effective treatment option for PSA that can mitigate the negative effects of the disease on HRQoL and productivity
\end{abstract}

Keywords Health-related quality of life $\cdot$ Intravenous golimumab $\cdot$ Productivity $\cdot$ Psoriatic arthritis

Alexis Ogdie

alexis.ogdie@uphs.upenn.edu

1 University of Pennsylvania, 3400 Spruce St, White Building, Room 5023, Philadelphia, PA 19104, USA

2 University of Utah, George E. Wahlen Veterans Affairs, Salt Lake City, UT, USA

3 Janssen Scientific Affairs, LLC, Horsham, PA, USA
4 Drexel University College of Medicine, Philadelphia, PA, USA

5 Janssen Global Services, LLC, Horsham, PA, USA

Janssen Research \& Development, LLC, Spring House, PA, USA

Janssen Global Services, LLC, Raritan, NJ, USA

University of California-San Diego, San Diego, CA, USA

9 Cleveland Clinic, Cleveland, OH, USA 


\section{Introduction}

Psoriatic arthritis (PsA) is a chronic inflammatory disease characterized by peripheral joint inflammation, enthesitis, dactylitis, spondylitis, psoriatic skin lesions, and nail psoriasis [1]. In addition to the multifaceted musculoskeletal and cutaneous burden, progressive joint deformity can result in significantly reduced health-related quality of life (HRQoL), functional impairment in performing daily and work-related activities, and disability [2-7]. Functional impairment can have substantial socioeconomic implications and is an important outcome measure in PsA [7, 8].

In a longitudinal analysis conducted over 10 years, $72 \%$ of patients with PsA experienced disability [9]. Health-related limitations at work, including absenteeism and reduced effectiveness, have been reported in 16 to $49 \%$ of patients with psoriasis or PsA [10-12]. In one report, work productivity, as measured by the Work Limitations Questionnaire (WLQ), was reduced by $7 \%$ in patients with PsA compared with benchmark employees without limitations [10]. In another multinational, real-world PsA population, the presence of enthesitis, dactylitis, inflammatory back pain, or sacroiliitis was significantly associated with worse patient quality of life and/or work productivity as measured by the EuroQol-5 dimension-5 level (EQ-5D-5L) index and visual analog scale (EQ-VAS) and the work productivity and activity index [13]. Thus, an important goal of PsA treatment is improved HRQoL and productivity.

In GO-VIBRANT, a Phase 3 randomized, placebocontrolled trial, intravenous (IV) golimumab, a fully human monoclonal antitumor necrosis factor (TNF) antibody, was both safe and efficacious, with sustained improvements in both joint and skin symptoms through 1 year in patients with active PsA [14, 15]. Additionally, improvements in physical function and HRQoL, evaluated using the Health Assessment Questionnaire-Disability Index (HAQ-DI), Short Form 36 Health Survey (SF-36) physical component summary (PCS) and mental component summary (MCS), EQ-VAS, Functional Assessment of Chronic Illness Therapy-Fatigue (FACIT-F), and Dermatology Life Quality Index (DLQI), were greater with golimumab versus placebo as early as Week 8 and through Week 24 [16]. Further, IV golimumab treatment has been shown to improve HRQoL, as well as work productivity in patients with the related spondyloarthritis, ankylosing spondylitis [17].

The aim of this post hoc analysis was to examine the long-term effects of IV golimumab on patient-reported outcome measures evaluating HRQoL (EQ-5D-5L index and EQ-VAS) through 1 year in patients with active PsA who participated in the GO-VIBRANT trial and to also evaluate changes in daily and work productivity in these patients. Additionally, we examined whether improvement in measures of HRQoL and productivity were associated with underlying improvement in measures of overall disease activity and patient functional capability.

\section{Materials and methods}

\section{Patients}

Details of the eligibility criteria and trial design of the GOVIBRANT trial were described previously [14]. Briefly, biologic-naïve patients $\geq 18$ years of age diagnosed with PsA for at least 6 months at screening based on ClASsification criteria for Psoriatic Arthritis (CASPAR) criteria [18] were included in the GO-VIBRANT trial. Patients had active PsA, defined as $\geq 5$ swollen and $\geq 5$ tender joints at screening and baseline and a C-reactive protein (CRP) level $\geq 0.6 \mathrm{mg} / \mathrm{dL}$ at screening, despite current or previous treatment with disease-modifying antirheumatic drugs ( $\geq 3$ months) and/or nonsteroidal antiinflammatory drugs ( $\geq 4$ weeks). All patients provided written informed consent.

\section{Trial design}

Eligible patients were randomized to receive IV golimumab 2 $\mathrm{mg} / \mathrm{kg}$ at Weeks 0 and 4 and then every 8 weeks (q8w) through Week 52 or placebo (normal saline for IV infusion) at Weeks 0 and 4 and then q8w, with crossover to IV golimumab $2 \mathrm{mg} / \mathrm{kg}$ at Weeks 24 and 28 and then $\mathrm{q} 8 \mathrm{w}$ through Week 52 [14]. At Week 16, patients in either treatment group who qualified for early escape $(<5 \%$ improvement in swollen and tender joint counts) were allowed to receive a protocol-specified change in concomitant medications at the investigator's discretion.

This trial was registered with clinicaltrials.gov (NCT02181673). The trial protocol was approved by an institutional review board or local ethics committee for each site, and the trial was conducted in accordance with the principles of the Declaration of Helsinki that are consistent with Good Clinical Practices and local regulatory requirements.

\section{Trial assessments}

General health status was measured using the United States model of the EQ-5D-5L standardized measure of health status, which is a descriptive system that comprises 5 dimensions-mobility, self-care, usual activities, pain/discomfort, and anxiety/depression [19]. Each dimension has 5 levels: no problems, slight problems, moderate problems, severe problems, and extreme problems. Each respondent was asked to indicate their health state in 
each of the 5 individual dimensions; a decrease in dimension score indicates improvement. The dimension scores were converted into a single summary index (EQ-5D-5L index) by applying a formula that attaches values (also called weights) to all possible combinations of levels in each dimension [20, 21]. The resulting score represents overall utility or general HRQoL, with 1 representing perfect health, 0 representing death, and negative values representing a state worse than death. The EQ-5D-5L also has a VAS element (EQ-VAS), which records the respondent's answer to the question "How good or bad is your health today?" on a vertical VAS where the endpoints are labeled "worst imaginable health state" $(0)$ and "best imaginable health state" (100); an increase in score indicates improvement.

Health-related productivity loss was evaluated using a daily productivity VAS and the WLQ. The daily productivity VAS records the respondent's answer to the question "How much has your disease affected your daily productivity at work, school, or home in the past 4 weeks" on a horizontal VAS where the endpoints are labeled "did not affect my productivity at all" (scored as 0 ) and "affected my productivity very much" (scored as 10); a decrease in score indicates improvement. The WLQ, which was administered only to patients who were working full or part time, including volunteering, is a 25 -item self-report questionnaire that asks respondents to rate their level of difficulty or ability to perform specific job demands [22]. The 25 items are aggregated into 4 domains - time management, physical demands, mentalinterpersonal, and output. Scale scores for each domain range from 0 (limited none of the time) to 100 (limited all of the time); a decrease in domain score indicates improvement. The 4 domains were also converted into an estimate of productivity loss (WLQ productivity loss score); a decrease in score indicates improvement.

Disease activity was assessed using the Psoriasis Area and Severity Index (PASI) [23], a modified version of the Disease Activity Score including 28 joints (DAS28) [24], and Disease Activity index for PSoriatic Arthritis (DAPSA) [25]. DAS28 is a statistically derived index combining 4 disease assessments (tender joint count [TJC; 28 joints], swollen joint count [SJC; 28 joints], CRP, and Patient's Global Assessment [PGA] of Disease Activity) that has historically been used in PsA clinical trials; however, DAPSA is a more valid measure of disease activity in patients with PsA as it includes the assessment of more joints (i.e., TJC 68 joints and SJC 66 joints) [25]. DAPSA also includes CRP, PGA of disease activity, and PGA of pain. Patient functional capability was assessed using the HAQ-DI [26] and the SF-36 PCS [27]. Mental health was assessed using the SF-36 MCS [27]. For PASI, DAS28, DAPSA, and HAQ-DI, a decrease in score indicates improvement [23-26]. For SF-36 MCS and PCS, an increase in score indicates improvement [27].

\section{Statistical analyses}

\section{Change in HRQoL and productivity measures by treatment group (discrimination)}

Prespecified analyses that included all randomized patients were changed from baseline through Week 52 by treatment group for EQ-5D-5L index, EQ-VAS, daily productivity VAS, and WLQ productivity loss score. Change from baseline in EQ-5D-5L index, EQ-5D-5L dimension scores, EQVAS, daily productivity VAS, WLQ productivity loss score, and WLQ domain scores by baseline methotrexate (MTX) use through Week 52 were post hoc analyses that included all randomized patients. Treatment group comparisons for EQ-5D-5L index, EQ-VAS, daily productivity VAS, and WLQ productivity loss score at Weeks 14 and 24 were prespecified; all other treatment group comparisons were unplanned. Unadjusted $p$ values of least squares mean differences between treatment groups through Week 24 were based on analysis of covariance, controlling for baseline score and baseline MTX usage. No formal comparisons were performed for time points after Week 24, when patients in the placebo group crossed over to golimumab, and there was no control group.

EQ-5D-5L index and dimension scores and EQ-VAS scores were based on observed data. Scores for daily productivity VAS, WLQ domains and productivity loss, PASI, DAS28, DAPSA, HAQ-DI, and SF-36 PCS and MCS were based on imputed data using last observation carried forward for missing data.

\section{Correlation of change in HRQoL and productivity measures with change in disease activity and patient functional capability measures in the IV golimumab group}

In patients randomized to the IV golimumab treatment group, Pearson correlation coefficient tests with Fisher's transformed 95\% confidence intervals were performed post hoc to evaluate the relationship between improvement from baseline in HRQoL (EQ-5D-5L index and EQ-VAS) and productivity outcomes (daily productivity VAS and WLQ productivity loss score) with underlying improvements in disease activity (PASI, DAS28, and DAPSA), patient functional capability (HAQ-DI and SF-36 PCS), and mental health (SF-36 MCS) outcomes.

\section{Impact of clinical outcomes on patient utility}

A multivariate analysis using a mixed-effect repeated measures model based on observed data until Week 24 (the 
placebo-controlled period) in the pooled patient population including both treatment groups was also conducted to quantify the impact of multiple attributes (age, gender, geographic region, PsA disease duration, PASI score, enthesitis, dactylitis, TJC 68, SJC 66, CRP, and HAQ-DI; independent variables) on utility, as measured by the EQ-5D-5L index (dependent variable). The independent variables were identified based on core outcome measures recommended by Outcome Measures in Rheumatology Clinical Trials (OMERACT) [7] and guideline utility mapping by the International Society for Pharmacoeconomics and Outcome Research (ISPOR) [28] and were included in the multivariate analysis based on univariate analyses and evaluation of collinearity between variables. Univariate analysis was first performed using a mixed-effect repeated measures model based on observed data until Week 24 in the pooled patient population to assess for association of attributes with the EQ-5D-5L index score. Variables were assessed for multicollinearity by variance inflation factor (VIF). A VIF of $<5$ was deemed acceptable [29]. Based on univariate analyses $(p<0.20)$ and evaluation of collinearity between variables, all of the previously listed attributes were included in the multivariate models. In the multivariate analysis, attributes were dropped based on statistical significance to yield the most parsimonious model. Akaike information criterion (AIC) was calculated for all models. AIC is a measure based on in-sample fit to estimate the likelihood of a model to predict or estimate the future values. A smaller AIC reflects a better fit.

\section{Results}

\section{Patient disposition and disease characteristics}

A total of 480 patients were randomized to IV golimumab $(n=241)$ or placebo $(n=239)$ (Table 1). Patient disposition through Week 52 has been previously reported in detail [15]. Baseline patient demographic and disease characteristics were generally well balanced between treatment groups (Table 1). Mean age was 46 years, and $52 \%$ of all patients were men. The EQ-5D-5L index score was 0.6 in both groups, EQ-VAS was 46.2 in the placebo group and 46.9 in the golimumab group, daily productivity VAS was 5.9 in the placebo group and 6.1 in the golimumab group, and the WLQ productivity loss score was 8.8 in the placebo group and 9.3 in the golimumab group.

\section{Change in HRQoL and productivity measures}

\section{EQ-5D-5L}

As early as Week 8 and through Week 24 , patients randomized to IV golimumab had greater mean improvements in the
EQ-5D-5L index than patients randomized to placebo (Week 8: 0.14 vs 0.04 , respectively; Week $24: 0.16$ vs 0.04 , respectively) (Fig. 1a). At Week 52, after patients randomized to placebo had crossed over to IV golimumab for several months, the golimumab group and the placebo-crossover group had similar mean improvements from baseline $(0.17$ and 0.15 , respectively). Results for the individual EQ-5D-5L dimension scores (i.e., mobility, self-care, usual activities, pain/discomfort, and anxiety/depression) were generally similar to those observed for the EQ-5D-5L index score, with greater mean improvements in each dimension score in the golimumab group versus the placebo group through Week 24 and similar improvement at Week 52 in the golimumab and placebo-crossover groups (Online Resource 1 Supplemental Fig. 1a-e).

\section{EQ-VAS}

Greater improvements in mean EQ-VAS scores in the IV golimumab group compared with the placebo group were also observed as early as Week 8 (17.16 vs 3.69 , respectively) and maintained through Week 24 (20.22 vs 5.51, respectively; $p<0.001$ ) (Fig. 1b). At Week 52, improvement in EQ-VAS score from baseline was similar between the golimumab and the placebo-crossover groups (21.61 and 20.84, respectively).

\section{Daily productivity VAS}

Improvements in mean daily productivity VAS scores were greater in patients randomized to IV golimumab than in patients randomized to placebo as early as Week $8(-2.91$ vs -0.71 , respectively) and maintained through Week 24 $(-3.33$ vs -0.89 , respectively; $p<0.001)$ (Fig. 2$)$. At Week 52 , improvements in mean daily productivity VAS scores were similar between the golimumab and placebo-crossover groups ( -3.31 and -2.89 , respectively).

\section{Work Limitations Questionnaire}

Among the 219 (46\%) patients (108 in the placebo group, 111 in the IV golimumab group) who were working or volunteering full or part time at baseline, improvements in mean WLQ productivity loss scores were greater in patients randomized to IV golimumab than in patients randomized to placebo as early as Week 8 ( -2.92 vs -0.78 , respectively) and maintained through Week 24 ( -4.04 vs -0.98 , respectively, $p<0.001$ ) (Fig. 3). At Week 52, improvements in mean WLQ productivity loss scores were similar between the golimumab and placebo-crossover groups $(-4.49$ and -3.28 , respectively). Results for the individual WLQ domain scores (i.e., mental-interpersonal, output, physical demands, and time management) were comparable to those observed for the WLQ productivity loss score, with greater mean improvements in 
Table 1 GO-VIBRANT baseline demographics and disease characteristics of randomized patients

\begin{tabular}{|c|c|c|c|}
\hline & Placebo & IV golimumab $2 \mathrm{mg} / \mathrm{kg}$ & Total \\
\hline Randomized patients, $n$ & 239 & 241 & 480 \\
\hline Age, years & $46.7(12.5)$ & $45.7(11.3)$ & $46.2(11.9)$ \\
\hline Male, $n(\%)$ & $121(50.6)$ & $128(53.1)$ & $249(51.9)$ \\
\hline \multicolumn{4}{|l|}{ Race, $n(\%)$} \\
\hline White & $237(99.2)$ & $241(100)$ & $478(99.6)$ \\
\hline Weight, kg & $82.8(17.9)$ & $84.4(21.1)$ & $83.6(19.6)$ \\
\hline BMI, $\mathrm{kg} / \mathrm{m}^{2}$ & $28.9(6.2)$ & $28.9(6.4)$ & $28.9(6.3)$ \\
\hline Duration of PsA, years & $5.3(5.9)$ & $6.2(6.0)$ & $5.8(6.0)$ \\
\hline Methotrexate use, $n(\%)$ & $173(72.4)$ & $163(67.6)$ & $336(70.0)$ \\
\hline SF-36 PCS, $0-100^{\mathrm{a}}$ & $34.0(7.2)$ & $33.1(6.9)$ & $33.6(7.1)$ \\
\hline SF-36 MCS, $0-100^{\mathrm{a}}$ & $42.5(10.2)$ & $43.5(11.4)$ & $43.0(10.8)$ \\
\hline HAQ-DI, 0-3 & $1.3(0.6)$ & $1.3(0.6)$ & $1.3(0.6)$ \\
\hline $\mathrm{CRP}, \mathrm{mg} / \mathrm{dL}$ & $2.0(2.1)$ & $1.9(2.5)$ & $2.0(2.3)$ \\
\hline Number of swollen joints, $0-66$ & $14.1(8.2)$ & $14.0(8.4)$ & $14.0(8.3)$ \\
\hline Number of tender joints, $0-68$ & $26.1(14.4)$ & $25.1(13.8)$ & $25.6(14.1)$ \\
\hline DAPSA $^{\mathrm{a}}$ & $54.9(22.8)$ & $54.2(21.6)$ & $54.6(22.2)$ \\
\hline DAS28 (CRP) ${ }^{\mathrm{a}}$ & $5.5(1.1)$ & $5.4(1.0)$ & $5.5(1.0)$ \\
\hline PASI score, $0-72^{\mathrm{b}}$ & $8.9(9.0)$ & $11.0(9.9)$ & $9.9(9.5)$ \\
\hline EQ-5D-5L Index, 0 to $1^{\mathrm{a}, \mathrm{c}}$ & $0.6(0.1)$ & $0.6(0.1)$ & $0.6(0.1)$ \\
\hline EQ-VAS, $0-100 \mathrm{~mm}^{\mathrm{a}}$ & $46.2(20.3)$ & $46.9(20.1)$ & $46.6(20.2)$ \\
\hline Daily productivity VAS, $0-10 \mathrm{~cm}^{\mathrm{a}}$ & $5.9(2.7)$ & $6.1(2.6)$ & $6.0(2.6)$ \\
\hline WLQ productivity loss score, $0-100 \mathrm{~mm}^{\mathrm{d}}$ & $8.8(4.7)$ & $9.3(5.2)$ & $9.0(5.0)$ \\
\hline
\end{tabular}

All values are mean (standard deviation) unless otherwise noted.

${ }^{\text {a }}$ Placebo $n=236$, IV golimumab $2 \mathrm{mg} / \mathrm{kg} n=237$, total $n=473$

${ }^{\mathrm{b}}$ Among patients with $\geq 3 \%$ BSA psoriasis skin involvement at baseline; placebo $n=188$, IV golimumab $2 \mathrm{mg} / \mathrm{kg}$ $n=189$, total $n=377$

${ }^{\mathrm{c}}$ Patients with severe disease may have values slightly below 0 , representing a state they believe is worse than death

${ }^{\mathrm{d}}$ Among patients who were working full or part time at baseline, including volunteering; placebo $n=108$, IV golimumab $2 \mathrm{mg} / \mathrm{kg} n=111$, total $n=219$

$B M I$, body mass index; $B S A$, body surface area; $C R P$, C-reactive protein; DAPSA, Disease Activity index for PSoriatic Arthritis; $D A S 28$, Disease Activity Score including 28 joints; EQ-5D-5L, EuroQol-5 dimension-5 level; $E Q-V A S$, EQ-5D-5L visual analog scale; $H A Q-D I$, Health Assessment Questionnaire-Disability Index; $I V$, intravenous; $M C S$, mental component summary; $n$, number of patients; PASI, Psoriasis Area and Severity Index; PCS, physical component summary; $P S A$, psoriatic arthritis; $S F-36$, Short Form 36 Health Survey; VAS, visual analog scale; $W L Q$, Work Limitations Questionnaire each domain score in the golimumab group versus the placebo group through Week 24 and similar improvement at Week 52 in the golimumab and placebo-crossover groups (Online Resource 1 Supplemental Fig. 2a-d).

\section{Baseline methotrexate use}

Overall, $70 \%$ of patients in this trial were using MTX at baseline $(72 \%$ in the placebo group and $68 \%$ in the golimumab group). Results were similar for all assessments at each time point regardless of baseline MTX use, with a few exceptions (Online Resource 1 Supplemental Figs. 1-3). For the EQ-5D$5 \mathrm{~L}$ anxiety/depression dimension score, in the golimumab group, the change from baseline through Week 52 was smaller in patients without versus with MTX use at baseline and, at Week 8, the change from baseline in the placebo group was smaller in patients with $(-0.05)$ versus without $(-0.17)$ baseline MTX use (Online Resource 1 Supplemental Fig. 1e). For EQ-VAS, in the placebo group, the change from baseline at Week 8 was smaller in patients with (2.22) versus without (7.23) MTX use at baseline (Online Resource 1 Supplemental Fig. 3b). For the WLQ mental-interpersonal domain score, in the placebo group, the change from baseline through Week 24 was smaller in patients with versus without baseline MTX use, and, in the golimumab group, improvement at Week 52 was smaller in patients without $(-4.52)$ 


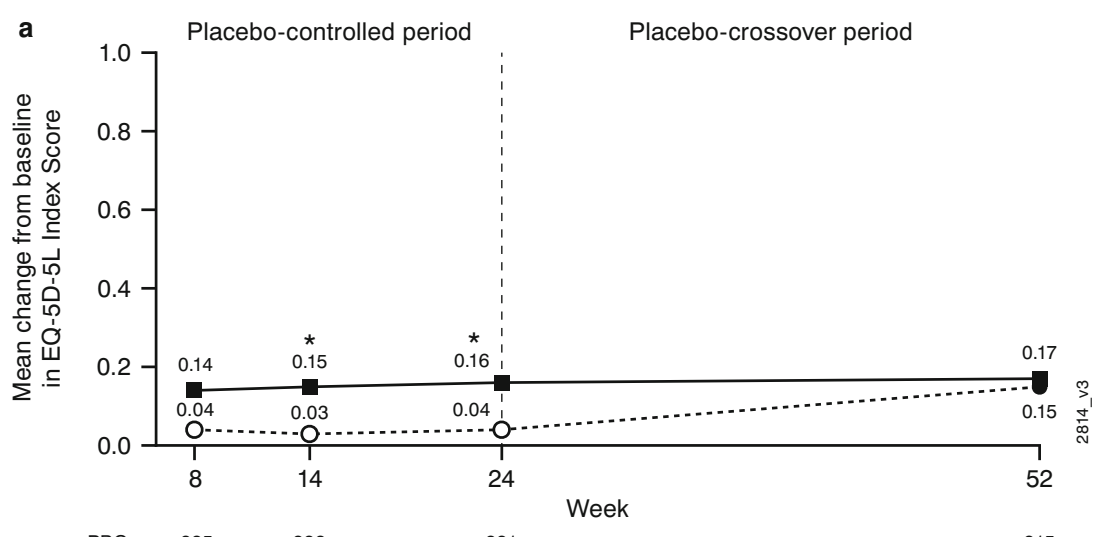

$\begin{array}{llll}\text { PBO } \mathrm{n}=225 & 222 & 221 & 215 \\ \text { GLM } \mathrm{n}=232 & 233 & 231 & 218\end{array}$

*LSMD: $0.12, p<0.001$

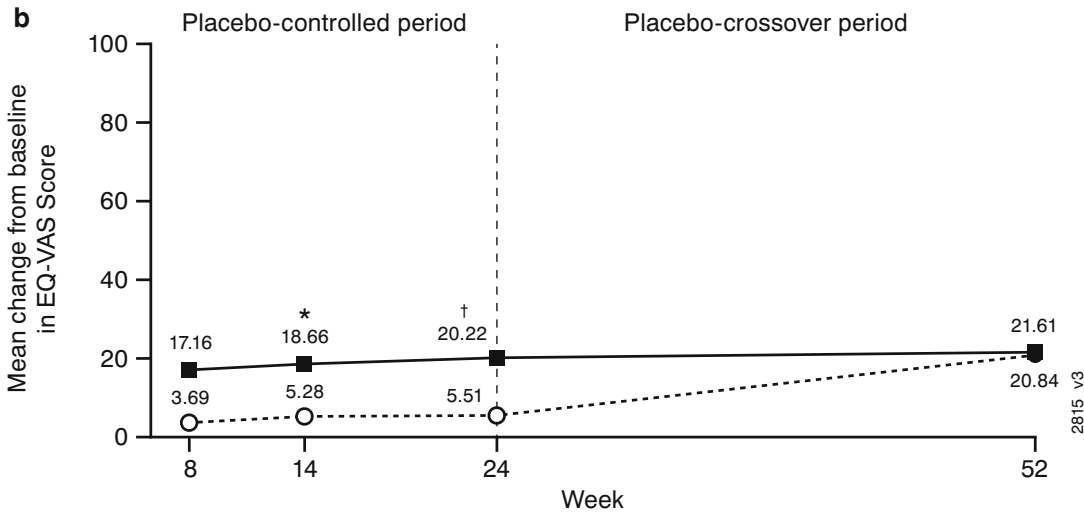

$\begin{array}{lll}\text { PBO } n=225 & 222 & 221\end{array}$

*LSMD: 13.65, $p<0.001 ;$ tLSMD: $15.01, p<0.001$

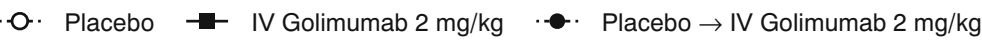

Fig 1 Mean change from baseline in EQ-5D-5L index (a) and EQ-VAS (b) scores through Week 52 in all randomized patients. Change from baseline is based on observed values. The adjusted $p$ values are based on ANCOVA controlling for baseline MTX usage (yes, no) and baseline EQ-5D-5L index (a) or EQ-VAS (b) scores.

ANCOVA, analysis of covariance; EQ-5D-5L, EuroQol-5 dimension-5 level; EQ-VAS, EQ-5D-5L visual analog scale; GLM, golimumab; $I V$, intravenous; $L S M D$, least square mean difference; $M T X$, methotrexate; $n$, number of patients; $P B O$, placebo

versus with (-13.52) baseline MTX use (Online Resource 1 Supplemental Fig. 2a). For this domain score, in patients without baseline MTX use, the change from baseline at Week 8 was greater in the placebo group compared with the golimumab group. Finally, for the WLQ output domain score, in the placebo group, improvement was smaller through Week 24 in patients with versus without baseline MTX use (Online Resource 1 Supplemental Fig. 2b).

\section{Correlation of change in HRQoL and productivity measures with disease activity and patient functional capability measures}

Improvements from baseline in the EQ-5D-5L index, EQ-VAS, WLQ productivity loss, and daily productivity VAS scores were generally moderately correlated with improvements from baseline in DAPSA, DAS28, HAQ-DI, and SF-36 PCS and MCS (Table 2, Online Resource 1 Supplemental Table 1). Correlations were generally similar from Week 8 through Week 52; however, EQ-VAS had weaker correlations with disease activity and functional capability measures at Weeks 8 and 14. DAS28 generally had slightly stronger correlations with HRQoL and productivity measures compared with DAPSA. Improvements in PASI weakly correlated with improvements in all of the HRQoL and productivity measures.

\section{Impact of clinical outcomes on the EQ-5D-5L index}

VIFs for all attributes originally included in this analysis (i.e., age, gender, geographic region, PsA disease duration, 


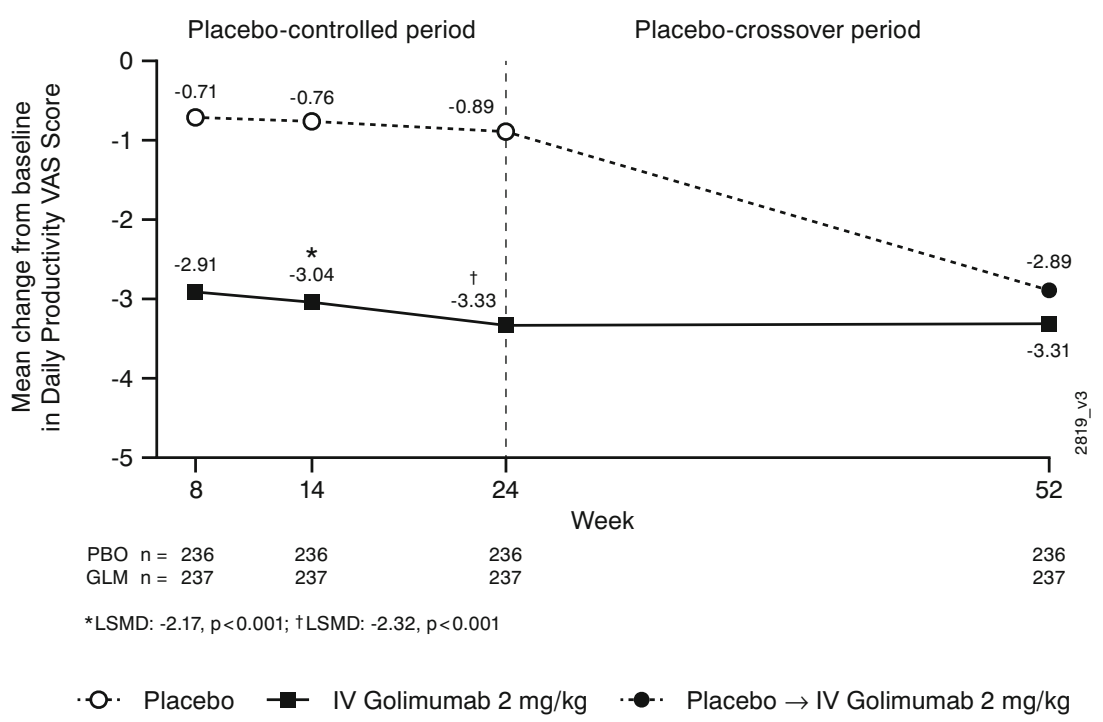

Fig 2 Mean change from baseline in daily productivity VAS score through Week 52 in all randomized patients. Change from baseline is based on imputed data using LOCF for missing data. Patients with no value at baseline are excluded from the analysis.

The adjusted $p$ values are based on ANCOVA controlling for baseline MTX use (yes, no) and baseline daily productivity VAS score. ANCOVA,

PASI score, enthesitis, dactylitis, TJC 68, SJC 66, CRP, and HAQ-DI) were $\leq 2.75$; therefore, multicollinearity is not likely a concern (generally, a VIF $>4$ suggests multicollinearity). Since the univariate regressions showed that all attributes were associated with the EQ-5D-5L index score through Week 24 with a $p$ value $<0.20$, they were all included in the multivariate analysis. In the final analysis of covariance; GLM, golimumab; $I V$, intravenous; $L O C F$, last observation carried forward; $L S M D$, least square mean difference; $M T X$, methotrexate; $n$, number of patients; $P B O$, placebo; $V A S$, visual analog scale

multivariate model, PASI score, enthesitis, TJC, CRP, and HAQ-DI were statistically significantly associated with the EQ-5D-5L index through Week 24 (Table 3). Based on the coefficient of each attribute in the final model, the impact of a 10-unit change in PASI score on the EQ-5D-5L index was similar to the impact of a 10-unit change in TJC or the presence of enthesitis.

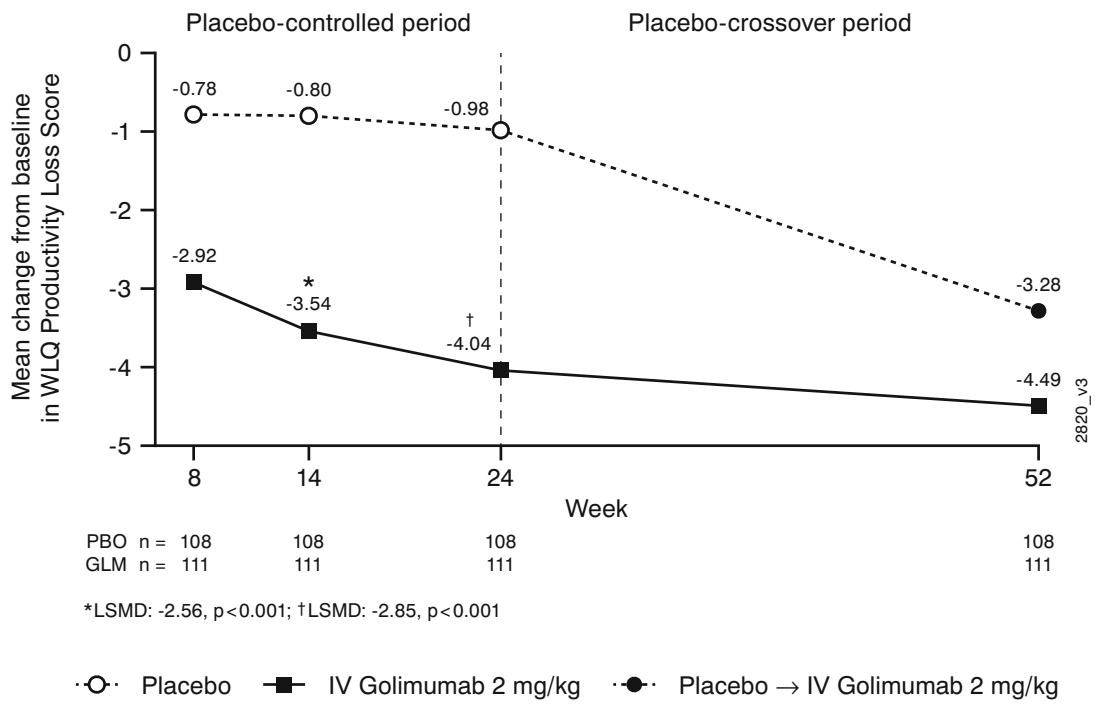

Fig 3 Mean change from baseline in WLQ productivity loss score through Week 52 among patients who were working or volunteering full or part time $(n=219)$. Change from baseline is based on imputed data using LOCF for missing data. Patients with no value at baseline are excluded from the analysis. The adjusted $p$ values are based on
ANCOVA controlling for baseline MTX usage (yes, no) and baseline WLQ productivity loss score. ANCOVA, analysis of covariance; $L O C F$, last observation carried forward; $L S M D$, least square mean difference; $G L M$, golimumab; $I V$, intravenous; $M T X$, methotrexate; $n$, number of patients; $P B O$, placebo; $W L Q$, Work Limitations Questionnaire 


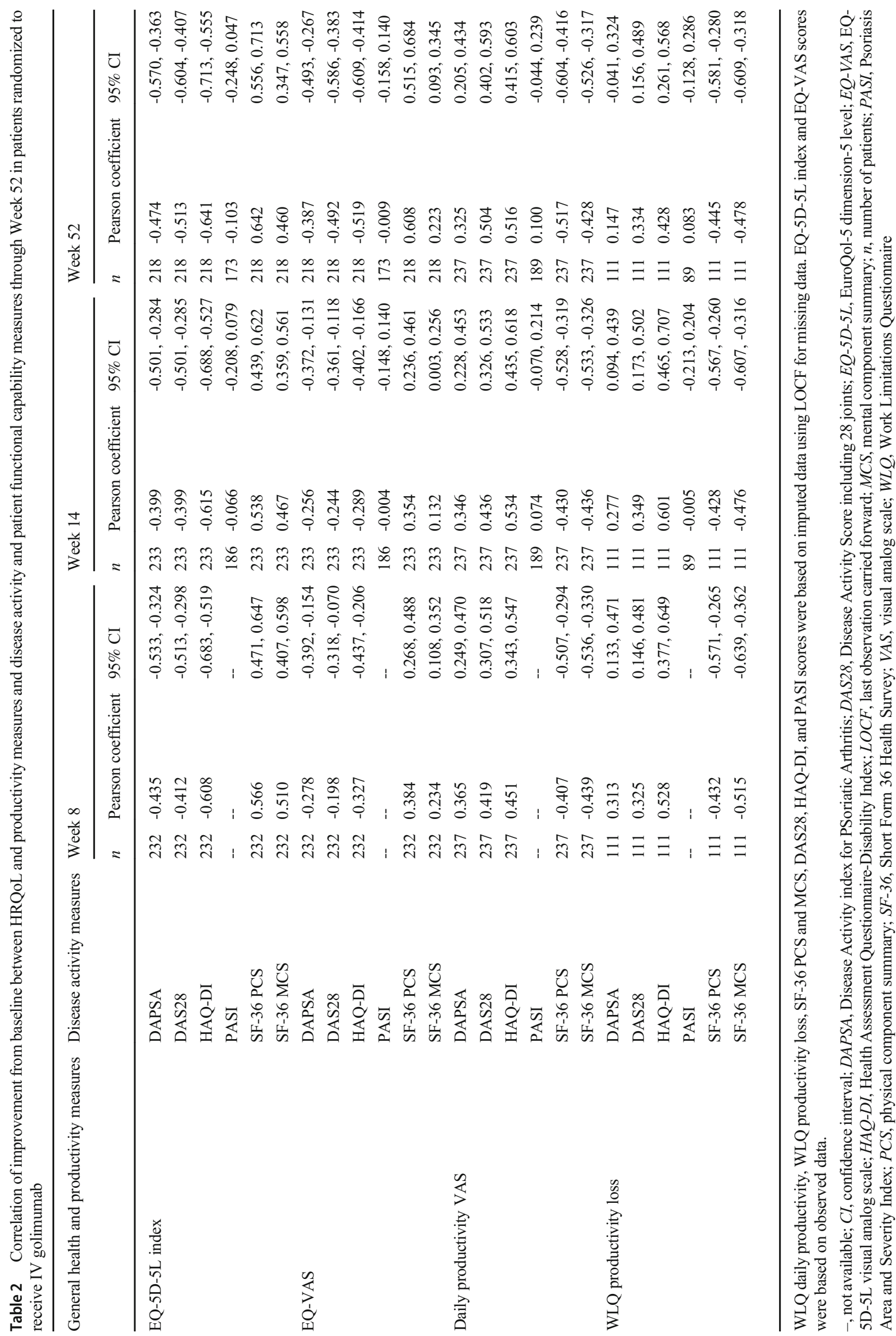


Table 3 Association of clinical manifestations of psoriatic arthritis with the EQ-5D-5L index in a multivariate mixed-effect repeated measures model

\begin{tabular}{|c|c|c|c|c|c|c|c|c|}
\hline \multirow[t]{2}{*}{ Variables } & \multicolumn{2}{|l|}{ Model 1} & \multicolumn{2}{|l|}{ Model 2} & \multicolumn{2}{|l|}{ Model 3} & \multicolumn{2}{|l|}{ Model 4} \\
\hline & $\beta$ & $p$ value & $\beta$ & $p$ value & $\beta$ & $p$ value & $\beta$ & $p$ value \\
\hline Age, years & 0.00030 & 0.3240 & 0.00031 & 0.2971 & 0.00031 & 0.2942 & & \\
\hline Female & -0.00215 & 0.7640 & -- & -- & -- & -- & -- & -- \\
\hline Geographic region (EU vs NA) & -0.02449 & 0.1981 & -0.02542 & 0.1780 & -0.02595 & 0.1678 & -- & -- \\
\hline Duration of PsA, years & 0.00016 & 0.7857 & -- & -- & -- & -- & -- & -- \\
\hline PASI score & -0.00123 & 0.0010 & -0.00122 & 0.0010 & -0.00121 & 0.0010 & -0.00126 & 0.0006 \\
\hline Enthesitis (yes vs no) & -0.01199 & 0.0424 & -0.01205 & 0.0412 & -0.01205 & 0.0398 & -0.01237 & 0.0348 \\
\hline Dactylitis (yes vs no) & -0.00083 & 0.8957 & -0.00073 & 0.9088 & -- & -- & -- & -- \\
\hline TJC (0-68) & -0.00122 & $<0.0001$ & -0.00122 & $<0.0001$ & -0.00116 & $<0.0001$ & -0.00112 & $<0.0001$ \\
\hline SJC (0-66) & 0.00017 & 0.6990 & 0.00016 & 0.7070 & -- & -- & -- & -- \\
\hline $\mathrm{CRP}(\mathrm{mg} / \mathrm{L})$ & -0.00078 & $<0.0001$ & -0.00078 & $<0.0001$ & -0.00077 & $<0.0001$ & -0.00079 & $<0.0001$ \\
\hline HAQ-DI & -0.16670 & $<0.0001$ & -0.16690 & $<0.0001$ & -0.16670 & $<0.0001$ & -0.1664 & $<0.0001$ \\
\hline \multicolumn{9}{|l|}{ Regression diagnostic } \\
\hline AIC & -2661.8 & & -2665.6 & & -2669.5 & & -2670.3 & \\
\hline
\end{tabular}

Based on 1367 observations.

AIC was similar for all models when attributes were omitted from the models.

-, not included in model; $A I C$, Akaike information criterion; $C R P$, C-reactive protein; EQ-5D-5L, EuroQol-5 dimension-5 level; EU, Europe; HAQ-DI, Health Assessment Questionnaire-Disability Index; NA, North America; PASI, Psoriasis Area and Severity Index; PsA, psoriatic arthritis; SJC, swollen joint count; TJC, tender joint count

\section{Discussion}

The data presented here demonstrate that reducing disease activity has a positive impact on HRQoL; daily productivity at work, school, or home; and productivity at work in patients with PsA that is maintained through 1 year. As expected based on previous results in this patient population [14-16], in patients with active PsA, treatment with IV golimumab resulted in improvements in EQ-5D-5L index, EQ-VAS, daily productivity VAS, and WLQ productivity loss score as early as Week 8 that were maintained through Week 52. Although the type of work performed by the patients who were working at baseline was not recorded in the GO-VIBRANT trial, it should be noted that greater improvement compared with placebo was observed in all domains of the WLQ, including the physical demands domain, suggesting that improvement in productivity would be observed across a wide variety of occupations. Consistent results have also been observed in other randomized controlled trials of biologics that have evaluated work productivity outcomes, including daily productivity VAS, the Work Productivity Survey, and the WLQ index, as well as absenteeism and presenteeism, in patients with PsA [8, $11,30,31]$.

These results are also consistent with those from similar analyses of data from a randomized placebo-controlled trial of IV golimumab in patients with ankylosing spondylitis [17], suggesting that IV golimumab is effective in improving HRQoL and productivity in a variety of spondyloarthritides. In patients with ankylosing spondylitis, greater improvements with IV golimumab versus placebo in EQ-5D-5L index, EQVAS, daily productivity VAS, WLQ, and the ankylosing spondylitis quality of life questionnaire (ASQoL) were observed as early as Week 8 through Week 16, and improvements were maintained with golimumab treatment through Week 52 [17]. The data reported here and the data from patients with ankylosing spondylitis also provide further support for clinical trial discrimination of the measures tested. The daily productivity VAS is not frequently used in clinical practice or randomized controlled trials, but may be a very useful instrument in clinical practice.

Improvements in this trial were generally similar among patients who were and were not receiving concomitant MTX at baseline. However, it should be noted that this trial was not designed to detect differences between golimumab + MTX and golimumab alone and possible associations between longer-term golimumab persistence and concomitant MTX use are unknown.

In this study and in the similar study in ankylosing spondylitis discussed previously [17], weak-to-moderate correlations of measures of HRQoL and productivity with measures of disease activity and patient functional capability, as assessed by the HAQ-DI, were observed, demonstrating that these domains are related, but that improvement is not completely mediated by measured symptoms alone. However, there may be benefits of suppression of TNF and reduction in systemic inflammation that may be measured by these HRQoL and productivity instruments that may not be 
fully reflected in clinical measures of disease activity and patient functionality alone.

Although correlations between improvement in PASI score and improvement in HRQoL and productivity measures were weak, our multivariate analysis showed that absolute PASI score was significantly associated with absolute EQ-5D-5L index score through Week 24. The multivariate analysis assessed association between absolute values and, therefore, is not bound by treatment effect, making it an inherently clearer measure of association between disease activity and EQ$5 \mathrm{D}-5 \mathrm{~L}$ index. In addition, correlation is not adjusted for scale and collinearity is not taken into account; thus, it is used here to 'eyeball' relationships between improvement in HRQoL and productivity measures and improvement in measures of disease activity and patient functionality, which in this particular trial, are bound by patient responses to IV golimumab.

A limitation of these data is that the results were observed in the context of a randomized controlled trial in patients with very high disease activity who predominantly have polyarticular disease. This may limit the generalizability of the observations to patients with less severe disease. Additionally, the type of work or hours of work per week may influence whether or not someone enrolls in a clinical trial; thus, there may be selection bias that should be considered when evaluating work outcomes. It should also be noted that the value set used for the EQ-5D-5L index score calculation in this study was based on the published United States value set for EQ-5D-5L [32]. Comparative exploration of differing EQ-5D-5L value sets by country may be explored in further analyses, but is beyond the scope of this current study. Further, the fact that the WLQ was only completed by patients who were working or volunteering at baseline does not allow for the evaluation of patients who may have started working following treatment during the study and should be considered when interpreting the WLQ results. The lack of information regarding whether patients were not working at baseline due to their disease and the type of work being done by patients who were working at baseline are also limitations of the trial.

In conclusion, PsA has a negative impact on HRQoL and productivity, and improvement in overall HRQoL and restoration of ability to engage productively in work and other activities remain an important goal of PsA treatment. In this trial, treatment with IV golimumab resulted in wide-ranging improvements in HRQoL and productivity, and these improvements appear to be associated with improvements in traditional measures of disease activity and patient functional capability.

Supplementary Information The online version contains supplementary material available at https://doi.org/10.1007/s10067-021-05639-1.

Acknowledgments Writing assistance was provided by Holly CapassoHarris of Synchrogenix, LLC, a Certara Company, on behalf of Janssen
Scientific Affairs, LLC and by Rebecca Clemente of Janssen Scientific Affairs, LLC.

Author contribution All authors contributed to the trial conception and design. Material preparation, data collection, and analysis were performed by Lilianne Kim, Kim Hung Lo, and Nan Li. All authors added significantly to the manuscript and read and approved the final manuscript.

Funding This work was supported by Janssen Research \& Development, LLC, Spring House, PA. Janssen Research \& Development LLC had a role in the trial design; collection, analysis, and interpretation of data; writing of the report; and the decision to submit the article for publication.

Data availability The data sharing policy of Janssen Pharmaceutical Companies of Johnson \& Johnson is available at https://www.janssen. $\mathrm{com} / \mathrm{clinical}$-trials/transparency. As noted on this site, requests for access to the trial data can be submitted through Yale Open Data Access (YODA) Project site at http://yoda.yale.edu.

Code availability Not applicable.

\section{Declarations}

Conflict of interest Alexis Ogdie has received consulting fees from Amgen, AbbVie Pharmaceuticals Inc., Bristol-Myers Squibb, Celgene, Corrona, Janssen, Eli Lilly and Company, Novartis, and Pfizer and has received grant support from Pfizer and Novartis (to University of Pennsylvania) and Amgen (to Forward Databank of which Dr. Ogdie is the principal investigator). Dr. Ogdie's husband has received royalties from Novartis. Jessica A. Walsh has received research grants or served as a consultant for AbbVie Pharmaceuticals Inc., Pfizer, Janssen, Eli Lilly and Company, Novartis, and UCB. Soumya D. Chakravarty is an employee of Janssen Scientific Affairs, LLC and owns stock in Johnson \& Johnson, of which Janssen Scientific Affairs, LLC is a wholly owned subsidiary. Steven Peterson, Nan Li, and Eric K.H. Chan are employees of Janssen Global Services, LLC and own stock in Johnson \& Johnson, of which Janssen Global Services, LLC is a wholly owned subsidiary. Kim Hung Lo, Lilianne Kim, and Elizabeth C. Hsia are employees of Janssen Research \& Development, LLC and own stock in Johnson \& Johnson, of which Janssen Research \& Development is a wholly owned subsidiary. Arthur Kavanaugh received grants from and/or served as a consultant for Amgen, Celgene, Eli Lilly and Company, Janssen, Pfizer, and UCB. M. Elaine Husni has served as a consultant for AbbVie Pharmaceuticals Inc., Amgen, Bristol-Meyers Squibb, Eli Lilly and Company, Gilead, Janssen, Novartis, Pfizer, Regeneron Pharmaceuticals, and UCB. The authors have full control of all primary data and agree to allow the journal to review the data if requested.

Open Access This article is licensed under a Creative Commons Attribution 4.0 International License, which permits use, sharing, adaptation, distribution and reproduction in any medium or format, as long as you give appropriate credit to the original author(s) and the source, provide a link to the Creative Commons licence, and indicate if changes were made. The images or other third party material in this article are included in the article's Creative Commons licence, unless indicated otherwise in a credit line to the material. If material is not included in the article's Creative Commons licence and your intended use is not permitted by statutory regulation or exceeds the permitted use, you will need to obtain permission directly from the copyright holder. To view a copy of this licence, visit http://creativecommons.org/licenses/by/4.0/. 


\section{References}

1. Gladman DD (2009) Psoriatic arthritis. Dermatol Ther 22:40-55

2. Husni ME, Merola JF, Davin S (2017) The psychosocial burden of psoriatic arthritis. Semin Arthritis Rheum 47:351-360

3. Kavanaugh A, Helliwell P, Ritchlin CT (2016) Psoriatic arthritis and burden of disease: patient perspectives from the populationbased multinational assessment of psoriasis and psoriatic arthritis (MAPP) survey. Rheumatol Ther 3:91-102

4. Kennedy M, Papneja A, Thavaneswaran A, Chandran V, Gladman DD (2014) Prevalence and predictors of reduced work productivity in patients with psoriatic arthritis. Clin Exp Rheumatol 32:342-348

5. Merola JF, Shrom D, Eaton J, Dworkin C, Krebsbach C, ShahManek B, Birt J (2019) Patient perspective on the burden of skin and joint symptoms of psoriatic arthritis: results of a multi-national patient survey. Rheumatol Ther 6:33-45

6. Rosen CF, Mussani F, Chandran V, Eder L, Thavaneswaran A, Gladman DD (2012) Patients with psoriatic arthritis have worse quality of life than those with psoriasis alone. Rheumatology 51 : 571-576

7. Orbai AM, de Wit M, Mease P, Shea JA, Gossec L, Leung YY, Tillett W, Elmamoun M, Duffin KC, Campbell W, Christensen R, Coates L, Dures E, Eder L, FitzGerald O, Gladman D, Goel N, Grieb SD, Hewlett S, Hoejgaard P, Kalyoncu U, Lindsay C, McHugh N, Shea B, Steinkoenig I, Strand V, Ogdie A (2017) International patient and physician consensus on a psoriatic arthritis core outcome set for clinical trials. Ann Rheum Dis 76:673-680

8. Iragorri N, Hofmeister M, Spackman E, Hazlewood GS (2018) The effect of biologic and targeted synthetic drugs on work- and productivity-related outcomes for patients with psoriatic arthritis: a systematic review. J Rheumatol 45:1124-1130

9. Husted JA, Tom BD, Farewell VT, Schentag CT, Gladman DD (2005) Description and prediction of physical functional disability in psoriatic arthritis: a longitudinal analysis using a Markov model approach. Arthritis Rheum 53:404-409

10. Walsh JA, McFadden ML, Morgan MD, Sawitzke AD, Duffin KC, Krueger GG, Clegg DO (2014) Work productivity loss and fatigue in psoriatic arthritis. J Rheumatol 41:1670-1674

11. Tillett W, de-Vries C, McHugh NJ (2012) Work disability in psoriatic arthritis: a systematic review. Rheumatology 51:275-283

12. Armstrong AW, Schupp C, Wu J, Bebo B (2012) Quality of life and work productivity impairment among psoriasis patients: findings from the National Psoriasis Foundation survey data 2003-2011. PLoS One 7:e52935

13. Walsh J, Ogdie A, Michaud K, Peterson S, Holdsworth E, Karyekar C, Booth N, Kemp J, Chakravarty S, Kafka S, Gossec L (2019) Enthesitis, dactylitis, and axial disease in psoriatic arthritis (PsA): impact on patient quality of life and work productivity [abstract]. Arthritis Rheum 71(suppl 10) https://acrabstracts.org/abstract/ enthesitis-dactylitis-and-axial-disease-in-psoriatic-arthritis-psaimpact-on-patient-quality-of-life-and-work-productivity/. Accessed 4 Oct 2019

14. Kavanaugh A, Husni ME, Harrison DD, Kim L, Lo KH, Leu JH, Hsia EC (2017) Safety and efficacy of intravenous golimumab in patients with active psoriatic arthritis: results through week twentyfour of the GO-VIBRANT study. Arthritis Rheum 69:2151-2161

15. Husni ME, Kavanaugh A, Murphy F, Rekalov D, Harrison DD, Kim L, Lo KH, Leu JH, Hsia EC (2020) Efficacy and safety of intravenous golimumab through 1 year in patients with active psoriatic arthritis. Arthritis Care Res (Hoboken) 72:806-813.

16. Husni ME, Kavanaugh A, Chan EKH, Peterson S, Li N, Hsia EC, Kim L, Lo KH, Harrison DD, Han C (2020) Effects of intravenous golimumab on health-related quality of life in patients with psoriatic arthritis: 24-week results of the GO-VIBRANT trial. Value Health 23:1286-1291

17. Reveille JD, Hwang MC, Danve A, Kafka S, Peterson S, Lo KH, Kim L, Hsia EC, Chan EKH, Deodhar A (2020) The effect of intravenous golimumab on health-related quality of life and work productivity in adult patients with active ankylosing spondylitis: results of the phase 3 GO-ALIVE trial. Clin Rheumatol. https:// doi.org/10.1007/s10067-020-05342-7 [Epub ahead of print]

18. Taylor W, Gladman D, Helliwell P, Marchesoni A, Mease P, Mielants H, CASPAR Study Group (2006) Classification criteria for psoriatic arthritis: development of new criteria from a large international study. Arthritis Rheum 54:2665-2673

19. EuroQol Group (1990) EuroQol - a new facility for the measurement of health-related quality of life. Health Policy 16:199-208

20. Shaw JW, Johnson JA, Coons SJ (2005) US valuation of the EQ$5 \mathrm{D}$ health states: development and testing of the $\mathrm{D} 1$ valuation model. Med Care 43:203-220

21. van Hout B, Janssen MF, Feng Y-S, Kohlmann T, Busschbach J, Golicki D, Lloyd A, Scalone L, Kind P, Pickard AS (2012) Interim scoring for the EQ-5D-5L: mapping the EQ-5D-5L to EQ-5D-3L value sets. Value Health 15:708-715

22. Lerner D, Amick BC III, Rogers WH, Malspeis S, Bungay K, Cynn D (2001) The work limitations questionnaire. Med Care 39:72-85

23. Fredriksson T, Pettersson U (1978) Severe psoriasis — oral therapy with a new retinoid. Dermatologica 157:238-244

24. van Riel PLCM (2014) The development of the disease activity score (DAS) and the disease active score using 28 joint counts (DAS28). Clin Exp Rheumatol 32(Suppl 85):S65-S74

25. Schoels M, Aletaha D, Funovits J, Kavanaugh A, Baker D, Smolen JS (2010) Application of the DAREA/DAPSA score for assessment of disease activity in psoriatic arthritis. Ann Rheum Dis 69:14411447

26. Fries JF, Spitz P, Kraines RG, Holman HR (1980) Measurement of patient outcome in arthritis. Arthritis Rheum 23:137-145

27. Ware JE Jr (2000) SF-36 health survey update. Spine (Phila Pa 1976) 25:3130-3139

28. Wailoo AJ, Hernandez-Alava M, Manca A, Mejia A, Ray J, Crawford B, Botteman M, Busschbach J (2017) Mapping to estimate health-state utility from non-preference-based outcome measures: an ISPOR good practices for outcomes research task force report. Value Health 20:18-27

29. Akinwande MO, Dikko HG, Samson A (2015) Variance inflation factor: as a condition for the inclusion of suppressor variable(s) in regression analysis. Open J Stat 5:754-767

30. Tillett W, Shaddick G, Jobling A, Askari A, Cooper A, Creamer P, Clunie G, Helliwell PS, James J, Kay L, Korendowych E, Lane S, Packham J, Shaban R, Thomas ML, Williamson L, McHugh N (2017) Effect of anti-TNF and conventional synthetic diseasemodifying anti-rheumatic drug treatment on work disability and clinical outcome in a multicenter observational cohort study of psoriatic arthritis. Rheumatology 56:603-612

31. Gniadecki R, Robertson D, Molta CT, Freundlich B, Pedersen R, Li W, Boggs R, Zbrozek AS (2012) Self-reported health outcomes in patients with psoriasis and psoriatic arthritis randomized to two etanercept regimens. J Eur Acad Dermatol Venereol 26:1436-1443

32. Pickard AS, Law EH, Jiang R, Pullenayegum E, Shaw JW, Xie F, Oppe M, Boye KS, Chapman RH, Gong CL, Balch A, Busschbach JJV (2019) United States valuation of EQ-5D-5L health states using an international protocol. Value Health 22:931-941

Publisher's note Springer Nature remains neutral with regard to jurisdictional claims in published maps and institutional affiliations. 